Published in final edited form as:

AIDS Behav. 2013 February ; 17(2): 508-516. doi:10.1007/s10461-012-0185-2.

\title{
Sexual Risk, Serostatus and Intimate Partner Violence Among Couples During Pregnancy in Rural South Africa
}

\author{
Karl Peltzer, \\ HIV/AIDS/STI and TB (HAST) Research Programme, Human Sciences Research Council, \\ Pretoria, South Africa \\ Department of Psychology, University of Limpopo, Turfloop, South Africa \\ Deborah Jones, \\ Department of Psychiatry and Behavioral Sciences, University of Miami Miller School of Medicine, \\ Miami, FL, USA
}

\section{Stephen M. Weiss,}

Department of Psychiatry and Behavioral Sciences, University of Miami Miller School of Medicine, Miami, FL, USA

\section{Olga Villar-Loubet, and}

Department of Psychiatry and Behavioral Sciences, University of Miami Miller School of Medicine, Miami, FL, USA

\section{Elisa Shikwane \\ HIV/AIDS/STI and TB (HAST) Research Programme, Human Sciences Research Council, Pretoria, South Africa}

Karl Peltzer: kpeltzer@hsrc.ac.za; Deborah Jones: djones@med.miami.edu; Stephen M. Weiss:

sweiss2@med.miami.edu; Olga Villar-Loubet: ovillar@med.miami.edu; Elisa Shikwane: eshikwane@hsrc.ac.za

\section{Abstract}

The aim of this study was to describe sexual risk behavior among 239 couples during pregnancy and to examine the relationship of sexual risk behavior with HIV serostatus and intimate partner violence. One-third $(31.8 \%)$ of pregnant women and $20.9 \%$ of male partners were HIV positive. HIV risk factors included lack of knowledge of partners' HIV serostatus, unprotected sexual intercourse and multiple sexual partners. Among men, multivariate logistic regression identified awareness of HIV negative partner status, multiple sexual partners and low levels of partner violence and among women Zulu or Swati ethnicity were associated with unprotected intercourse. HIV positive concordance was associated with protected sex and in multilevel analysis of couples HIV positive status and awareness of the partner's HIV positive status were associated with protected sex. High levels of HIV risk behaviour was found among couples during pregnancy calling for HIV risk reduction interventions. 


\section{Keywords}

Couples; Pregnant women; HIV status; HIV risk behavior; South Africa

\section{Background}

Though male to female transmission of HIV has been estimated as 2.3 times greater than female to male transmission [1,2], the risk of HIV acquisition rises for both men and women during pregnancy [3]. This change is likely to be due to both sexual risk behaviours and to hormonal changes affecting the genital tract mucosa or immune responses [3, 4]. In fact, previous studies found the increased HIV-1 risk (7.35 vs. 3.01 during HIV pregnant and non-pregnant periods) was attenuated after adjusting for sexual behavior and confounds [3]. However, the risk of female to male transmission was 3.46 versus 1.58 when comparing pregnant and non-pregnant female partners, the risk was not attenuated by behavior or confounds. Thus, elevated risk of HIV-1 acquisition during pregnancy appears to be explained by both behavioral and biological factors [3]. Finally, in addition to the risk of HIV transmission from mother to child, there is also an association between adverse pregnancy outcomes and HIV seropositive status (stillbirth, spontaneous abortion, OR 3.91, 4.05, respectively, [5]; infant mortality, OR 3.69, [6]).

Sexual intercourse frequency has been found to decrease in the third trimester of pregnancy [7-9]. For example, among Thai pregnant women during each trimester, most women had the coital frequency of $0-1$ times per week $(76.7,82.5$ and $92.5 \%$, respectively), followed by $2-3$ times a week $(18.3,16.7$ and $7.5 \%$, respectively) and more than 3 times a week (5, 0.8 and $0 \%$, respectively). However, in Cape Town, South Africa, while pregnant women had fewer male partners compared to non-pregnant women in the previous 6 months, they also reported less condom use and were less likely to request that their partners use condoms [10]. Similarly, in Rakai, Uganda, condom use was found to be less consistent during pregnancy [4]. In fact, among couples that associate condom use with contraception rather than risk reduction, sex may be more likely to be unprotected throughout pregnancy, greatly increasing the risk for HIV transmission, with women engaging in more HIV risk behavior overall [11]. Finally, among HIV serodiscordant or concordant couples, the desire to conceive may lead to risky behaviour despite knowledge of serostatus [12]. In Cape Town, over $30 \%$ of HIV positive women and $65 \%$ of HIV negative men attending public sector clinics reported an interest in having additional children, and in Johannesburg, $60 \%$ of HIV positive women had plans to conceive in the next year, while in both groups, most had never had a conversation with a health care worker on this issue [13]. Individuals may tend to overestimate their spouse's, as well as their own risk of having HIV [14]. While marital infidelity is the most important correlate of overestimation of individual and spousal HIV risk, during pregnancy, concerns regarding partners' HIV status may be superseded by the reduced need for contraception.

In sub-Saharan Africa, domestic physical violence has been associated with HIV infection [15], and a significant overlap exists between women who are seropositive and those who are battered [16]. The ability to negotiate safer sex is key to controlling transmission and perceived power within the relationship has been associated with condom use among 
women [17], and with condom decision making [18]. In fact, antenatal women with violent or controlling male partners were found to be at increased risk of HIV infection, and at risk of having risky sexual practices imposed upon them [19]. Transmission rates may be highest between primary partners [20].

The aim of this study was to describe sexual risk behavior among couples during pregnancy in rural South Africa, and to examine the relationship of sexual risk behavior with HIV serostatus and intimate partner violence.

\section{Methods}

\section{Sample and Procedure}

This study recruited 239 couples ( $\mathrm{n}=478$ individuals) from 12 community health centres (about 20 couples per clinic) in Nkangala and Gert Sibande health districts, Mpumalanga province, South Africa. Women participants were pregnant women (24-30 weeks gestation) age 18 and older who had received HIV counselling and testing (HCT) at the antenatal care clinic and men participants were aged 18 or older with an enrolled pregnant partner. At the antenatal care clinic, following HCT, participants were informed about the study and referred for participation if interested. Following initial contact with prospective participants, study staff obtained a medical and social (couple) history to determine initial eligibility for the study. Couples were interviewed separately for couple eligibility [21], and were asked a short series of rotating questions related to couple-specific issues e.g., "who sleeps next to the window? What colour is your blanket?" Staff compared responses to access couple responses and qualify or disqualify couples at this point. Couples meeting basic study criteria were invited to provide and sign the Informed Consent form. Participants then completed a baseline psychosocial and behavioural assessment. All questionnaire data were obtained by being read to the participants to eliminate potential confounds related to literacy. Participant assessments included both biological (clinic data) and psychosocial assessments. Participants received R100 for the assessment for time and transportation.

The study received ethical approval from the Human Sciences Research Council Research Ethics Committee (Protocol REC No. 1/18/08/10), the University of Miami Miller School of Medicine (Protocol No. 20100555), the National Institute of Allergies and Infectious Diseases and the Provincial Department of Health of Mpumalanga, South Africa.

\section{Measures}

\section{Biological Assessment}

Clinic data: Results of the HIV test for women and for male partners.

HIV knowledge was assessed with 12 items adapted from a reported measure [22, 23] and reflected information about HIV transmission, condom use, and AIDS-related knowledge and were responded to Yes, No, or Don't Know. The HIV knowledge test was scored for the number of correct responses, with Don't Know responses scored as incorrect $(=0)$, and the possible range of scores 0 to 12 expressed as the percentage correct. The HIV knowledge test demonstrated heterogeneous item content; Cronbach's alpha (a) was 0.54 for this study 
sample. The data from the HIV knowledge index were positively skewed which may explain the low consistency. The HIV knowledge index was recoded into low (scores 4-8), medium (scores 9-10) and high (scores 11-12).

Scales used in this study were modified using a three step process. First, each scale was reviewed by the entire team to assess the content validity of the items in the South African context, and to delete unnecessary items to reduce patient burden, e.g., item duplication between measures, items not relevant to the study aims. Next, each scale item was reviewed by the team for reliability, to ensure that the item was understandable, e.g., poorly worded items were deleted or revised to enhance clarity. Finally, the combined scales were tested with assessors from the local setting, who provided additional feedback on item clarity during role play assessments, e.g., items were revised that were confusing.

Sexual risk was assessed with a 16-item scale adapted from the Sexual Risk Behavior Assessment Schedule [24] which assessed the number of unprotected vaginal and anal intercourse occasions (i.e. with or without condom use) by partner type (main vs. non-main partners), number of partners, and gender of partners for 1-month prior to each assessment, using timeline-follow back type cues for recall. Cronbach a for this scale for women was 0.71 and for men 0.68 in this sample.

Sexual barrier use was assessed by sexual diary. Responses indicated for each day of the week occurrence of sexual intercourse and type of sexual barrier method used, if any.

Sexual diary data represented an assessment of sexual behavior and condom use over a 7 day period, while sexual risk behavior data captured a 1 month period assessing types of behavior (e.g., vaginal, anal sex, multiple partners). For the purposes of condom use, the 7 day data was selected due to the anticipation of more accurate recall.

Intimate partner violence was assessed using a modified 17-item version of the conflict tactics scale (CTS) [25]. The CTS measures the extent to which current or previous partners in a dating, cohabiting, or marital relationship have responded to conflict using negotiation, verbal aggression or violence. Negotiation is assessed with three items, e.g., "Discussed an issue calmly"; Verbal aggression is assessed with seven items, e.g., "Insulted or swore at him/her"; Minor violence is assessed with three items, e.g., "Throw something at him/her." Severe violence is assessed with six items, e.g., "Kicked, bit, or hit him/her with a fist" [26]. Response options ranged from $0=$ Never to $1=$ ever done in the last month; subscales were scored by adding each response category code value. Cronbach's alpha ( $a$ ) for the CTS was 0.87 for this study sample. For the purposes of the current study, the CTS was adapted to address only violence experience in the last month which related directly to the couple under consideration.

\section{Statistical analyses}

The IBM statistical software Statistical Package for Social Sciences (SPSS version 19.0 for Windows; Chicago, IL, USA) was used for data analyses. Pearson $\chi^{2}$ and Student $t$ tests were used for analysis of proportions. Bivariate analysis and multivariable logistic regressions were used to investigate associations between the predictor variables and the 
outcome, non-condom use (coded as 0,1 ). Non-condom use was calculated as not having had any protected sexual intercourse in the past 7 days, based on the sexual diary. The factors that were found to be significant in the bivariate analysis were first included into a full model. Associations were considered significant at $P<0.05$. In addition, due to the interdependence within dyads, mixed multilevel modelling (MLM) was used for the analyses unless otherwise specified, in which the condition or dyad was the upper level of the hierarchy in the model from which the predictor "condition" was drawn, and the individual was the lower level, from which the outcome variable was drawn ("unprotected sex") as well as the remaining predictors ("alcohol and drug use, intimate partner violence"). Categorical variables were effects coded (recoded as 1, -1 : serostatus, alcohol and drug use) and continuous variables were grand mean centered (the grand mean was subtracted from all predictor scores: violence) for MLM analyses.

\section{Results \\ Sample Characteristics}

The age of the study participants was on average 28.2 years $(\mathrm{SD}=7.1$ ), ranging from 18 to 53 years. Male partners were significantly older, better educated, employed, receiving a salary, and had a higher income than female partners. Most participants resided in rural areas $(71.1 \%)$, were Zulu and Ndebele ethnicity and had on average 1 child $(\mathrm{SD}=1.2)$ (see Table 1).

\section{Sexual Behaviour and HIV Serostatus}

Medical record abstraction confirmed that almost one-third (31.8\%) of the pregnant women were HIV positive, while $20.9 \%$ of their male partners were HIV positive, with approximately two-thirds acknowledging their serostatus to study assessors. Almost half of the women $(46.9 \%)$ were not aware of the HIV status of their male partner, while only 14.2 $\%$ of the men did not know the HIV status of their female partners. The average number of sexual acts in the previous week was $2.8(\mathrm{SD}=3.0), 51.9 \%$ of the male and $47.7 \%$ of the female partners had at least one incident of unprotected sexual intercourse in the past week, and $17.6 \%$ of the men and $10.0 \%$ of the women participants indicated that they had had sex with someone else (not their primary partner) in the past month. Almost one-third (27.7\%) of the men and $16.7 \%$ of the women had used alcohol before having sexual intercourse in the past month (see Table 2).

\section{Associations Between Unprotected Sex, HIV Serostatus, Sexual Partnering and Intimate Partner Violence}

In bivariate analysis among men of Swati ethnicity, being HIV negative, awareness of HIV negative partner status, having multiple sexual partners in the past month and low minor partner violence were associated with unprotected intercourse in the past week, while in multivariate unconditional logistic regression, awareness of HIV negative partner status, having multiple sexual partners in the past month and low minor partner violence were associated with unprotected intercourse in the past week. 
In bivariate analysis among women of Swati ethnicity, being HIV negative and awareness of HIV negative partner status were associated with unprotected intercourse in the past week, while in multivariable unconditional logistic regression only being of Zulu or Swati ethnicity were associated with unprotected intercourse in the past week. Regarding HIV concordance or discordance, among men, HIV positive concordance was inversely associated with unprotected sex, and among women, HIV positive concordance and discordance were inversely associated with unprotected sex (see Table 3).

Data was restructured to conduct dyadic comparisons of the independent variables with unprotected sex, using multilevel modeling. HIV positive status and awareness of the HIV positive status of the partner were inversely associated with unprotected intercourse (see Table 4).

\section{Discussion}

This study sought to describe sexual risk behavior among couples during pregnancy in rural South Africa and to examine the relationship of sexual risk behavior with HIV serostatus and intimate partner violence. Results indicate that among pregnant women (24-30 weeks gestation) and their male partners, sexual intercourse and HIV sexual risk behaviour was frequent, including unprotected sex, multiple partners and sexual partners of unknown serostatus. Previous studies found a much lower coital frequency in the last trimester $(92.5$ $\% 0-1$ times) [7] than that obtained in this study (on average 2.8 times). Regarding sexual risk, previous studies had similar findings related to increased HIV risk behaviour among pregnant women (e.g., less condom use among pregnant than non-pregnant women [10]; unprotected sex with partners who were HIV positive [11]). In the current study, men reported greater HIV risk behaviour (multiple sexual partners, alcohol use in the context of sex) than women, not unlike previous research [27].

Interestingly, this study found that unprotected sex to be higher among male partners who were aware of the HIV negative status of their female partner, who had had multiple sexual partners in the past month and those who experienced low levels of minor partner violence. While it may be understandable that male partners have unprotected sex with an HIV negative partner, it is disturbing that male partners with multiple sex partners also have more unprotected sex. The experience of minor partner violence among male partners seems to be protective in terms of condom use. In a study among pregnant women in rural Haiti, results showed that gender and power factors were most significant for condom use. These results suggest the need to create prevention interventions that restore power imbalances and strengthen communication skills [28]. Among women, those who were Swati or Zulu were at higher risk for unprotected intercourse than Ndebele and Northern Sotho pregnant women. This finding seems to concur with higher HIV prevalence rates among Swati and Zulu ethnicities as compared to Ndebele and Northern Sotho ethnic groups in South Africa [29]. Further, HIV positive concordance was associated with protected sex and in multilevel analysis of couples, HIV positive status and awareness of the HIV positive status of the partner were associated with protected sex. These findings are in concordance with other studies [30]. 
This study had several limitations. Firstly, the study used a purposeful sample. There may be differences between couples who chose to enroll in the study and those who did not. It could be that HIV positive participants did not participate because of stigma. Further, the selfreport of sexual behaviour should be interpreted with caution; it is possible that respondents underreported sexual risk behaviour, especially females. Furthermore, this study was based on data collected in a cross-sectional survey. We cannot, therefore, ascribe causality to any of the associated factors in the study. Prospective studies are required to confirm the sexual behaviour findings.

\section{Conclusion}

This study identified both ongoing violence and high levels of HIV risk behaviour among couples in rural South Africa during pregnancy. Recent studies have highlighted the heightened risk of HIV transmission during pregnancy for both sexual partners. This increased risk of HIV transmission and the burst of viremia associated with HIV infection make unprotected sex during pregnancy especially dangerous to mothers. Results of this study accentuate the need for targeted HIV risk reduction interventions for couples during pregnancy, including continued emphasis on HIV testing by both members of the couple, as well as couples counseling during pregnancy regarding the risks associated with unprotected sex. However, clinicians must remain cognizant of the danger of IPV in this population, and community interventions should be developed to reduce the incidence of IPV. Clearly, with the advent of effective ARV intervention, couples are increasingly likely to consider having children, and there is a growing public health need to address both sexual behavior during pregnancy as well as plans for future pregnancies in this vulnerable population.

\section{Acknowledgments}

The study is funded by the National Institute of Allergy and Infectious Diseases, grant number P30AI073961-S2.

\section{References}

1. Nicolosi A, Leite MLC, Musicco M, Arici C, Gavazzeni G, Lazzarin A. The efficiency of male-tofemale and female-to-male sexual transmission of the Human Immunodeficiency Virus: a study of 730 stable couples. Epidemiology. 1994; 5(6):570-575. [PubMed: 7841237]

2. Otwombe KN, Ndindi P, Ajema C, et al. Using VCT statistics from Kenya in understanding the association between gender and HIV. SAHARA J. 2007; 4(3):110-115.

3. Mugo NR, Heffron R, Donnell D, et al. Increased risk of HIV-1 transmission in pregnancy: a prospective study among African HIV-1-serodiscordant couples. AIDS. 2011; 25(15):1887-1895. [PubMed: 21785321]

4. Gray RH, Li X, Kigozi G, et al. Increased risk of incident HIV during pregnancy in Rakai, Uganda: a prospective study. Lancet. 2005; 366(9492):1182-1188. [PubMed: 16198767]

5. Desgrées du Loû A, Msellati P, Ramon R, et al. HIV-1 infection and reproductive history: a retrospective study among pregnant women, Abidjan, Côte d'Ivoire, 1995-1996. Ditrame Project. Int J STD AIDS. 1998; 9(8):452-456. [PubMed: 9702593]

6. Brocklehurst P, French R. The association between maternal HIV infection and perinatal outcome: a systematic review of the literature and meta-analysis. Br J Obstet Gynaecol. 1998; 105(8):836-848. [PubMed: 9746375]

7. Senkumwong N, Chaovisitsaree S, Rugpao S, et al. The changes of sexuality in Thai women during pregnancy. J Med Assoc Thail. 2006; 89(4):S124-S129. 
8. Pauleta JR, Pereira NM, Graça LM. Sexuality during pregnancy. J Sex Med. 2010; 7(1 Pt 1):136142. [PubMed: 19845548]

9. Bartellas E, Crane JM, Daley M, et al. Sexuality and sexual activity in pregnancy. Br J Obstet Gynaecol. 2000; 107(8):964-968.

10. Onoya D, Reddy P, Sifunda S, et al. Comparing STI risk and sexual behaviour profiles of pregnant versus non-pregnant, HIV negative black south African women. WebmedCentral PUBLIC HEALTH. 2010; 1(11) WMC001142.

11. Manenti SA, Galato JJ, Silveira ES, et al. Epidemiologic and clinical characteristics of pregnant women living with HIV/AIDS in a region of Southern Brazil where the subtype C of HIV-1 infection predominates. Braz J Infect Dis. 2011; 15(4):349-355. [PubMed: 21861005]

12. Brubaker SG, Bukusi EA, Odoyo J, et al. Pregnancy and HIV transmission among HIV-discordant couples in a clinical trial in Kisumu, Kenya. HIV Med. 2011; 12(5):316-321. [PubMed: 21205129]

13. Schwartz SR, Mehta SH, Taha TE, Rees HV, Venter F, Black V. High Pregnancy Intentions and Missed opportunities for Provider communication about fertility in a South African cohort of HIV positive women on antiretroviral therapy. AIDS Behav. 2011

14. Anglewicz PA, Bignami-Van Assche S, Clark S, et al. HIV risk among currently married couples in rural Malawi: What do spouses know about each other? AIDS Behav. 2010; 14(1):103-112. [PubMed: 19051003]

15. Ackermann L, de Klerk K. Social factors that make South African women vulnerable to HIV infection. Health Care Women Int. 2002; 3:163-172. [PubMed: 11868963]

16. Maman S, Mbwambo JK, Hogan NM, et al. HIV-positive women report more lifetime partner violence: findings from a voluntary counseling and testing clinic in Dar es Salaam, Tanzania. Am J Public Health. 2002; 92:1331-1552. [PubMed: 12144993]

17. Pettifor AE. Sexual power and HIV risk, South Africa. Emerg Infect Dis. 2004; 10(11):1996-2004. [PubMed: 15550214]

18. Harvey SM, Bird ST, Galavotti C, et al. Relationship power, sexual decision-making, and condom use among women at risk for HIV/STDs. Women Health. 2002; 36(4):69-84. [PubMed: 12555803]

19. Dunkle KL, Jewkes RK, Brown HC, et al. Gender-based violence, relationship power, and risk of HIV infection in women attending antenatal clinics in South Africa. Lancet. 2004; 363(9419): 1415-1421. [PubMed: 15121402]

20. Kalichman SC, Rompa D, Luke W, et al. HIV transmission risk behaviors among HIV-positive persons in serodiscordant relationships. Int J STD AIDS. 2002; 13(10):677-682. [PubMed: 12396537]

21. El-Bassel N, Witte SS, Gilbert L, et al. The efficacy of a relationship- based HIV/STD prevention program for heterosexual couples. Am J Public Health. 2003; 93(6):963-969. [PubMed: 12773363]

22. Carey MP, Schroder KE. Development and psychometric evaluation of the brief HIV knowledge questionnaire (HIV-KQ-18). AIDS Educ Prev. 2002; 14:174-184.

23. Peltzer K, Mlambo G, Phaweni K. Factors determining prenatal HIV testing for prevention of mother to child transmission of HIV in Mpumalanga, South Africa. AIDS Behav. 2010; 14(5): 1115-1123. [PubMed: 20049520]

24. Meyer-Bahlberg, HFL.; Ehrhardt, AA.; Exner, TM., et al. Sexual risk behavior assessment schedule: adult. (SERBAS-A-DF-4) manual. New York: Psychological Press; 1990.

25. Straus MA. Measuring intrafamily conflict and violence. J Marriage Fam. 1979; 41:75-88.

26. Strauss MA, Hamby SL, Boney-McCoy S. The revised conflict tactics scales (CTS2): development and preliminary psychometric data. J Fam Issues. 1996; 17(3):283-316.

27. Bennetts A, Shaffer N, Phophong P, et al. Differences in sexual behaviour between HIV-infected pregnant women and their husbands in Bangkok, Thailand. AIDS Care. 1999; 11(6):649-661. [PubMed: 10716006]

28. Kershaw TS, Small M, Joseph G, Theodore M, Bateau R, Frederic R. The influence of power on HIV risk among pregnant women in rural Haiti. AIDS Behav. 2006; 10(3):309-318. [PubMed: 16482404] 
29. Department of Health. National Antenatal Sentinel HIV and Syphilis Prevalence Survey in South Africa, 2010. Pretoria: Department of Health; 2011.

30. Mlambo M, Peltzer K. HIV sero-status disclosure and sexual behaviour among HIV positive patients who are on antiretroviral treatment (ART) in Mpumalanga, South Africa. J Hum Ecol. 2011; 35(1):29-41. 


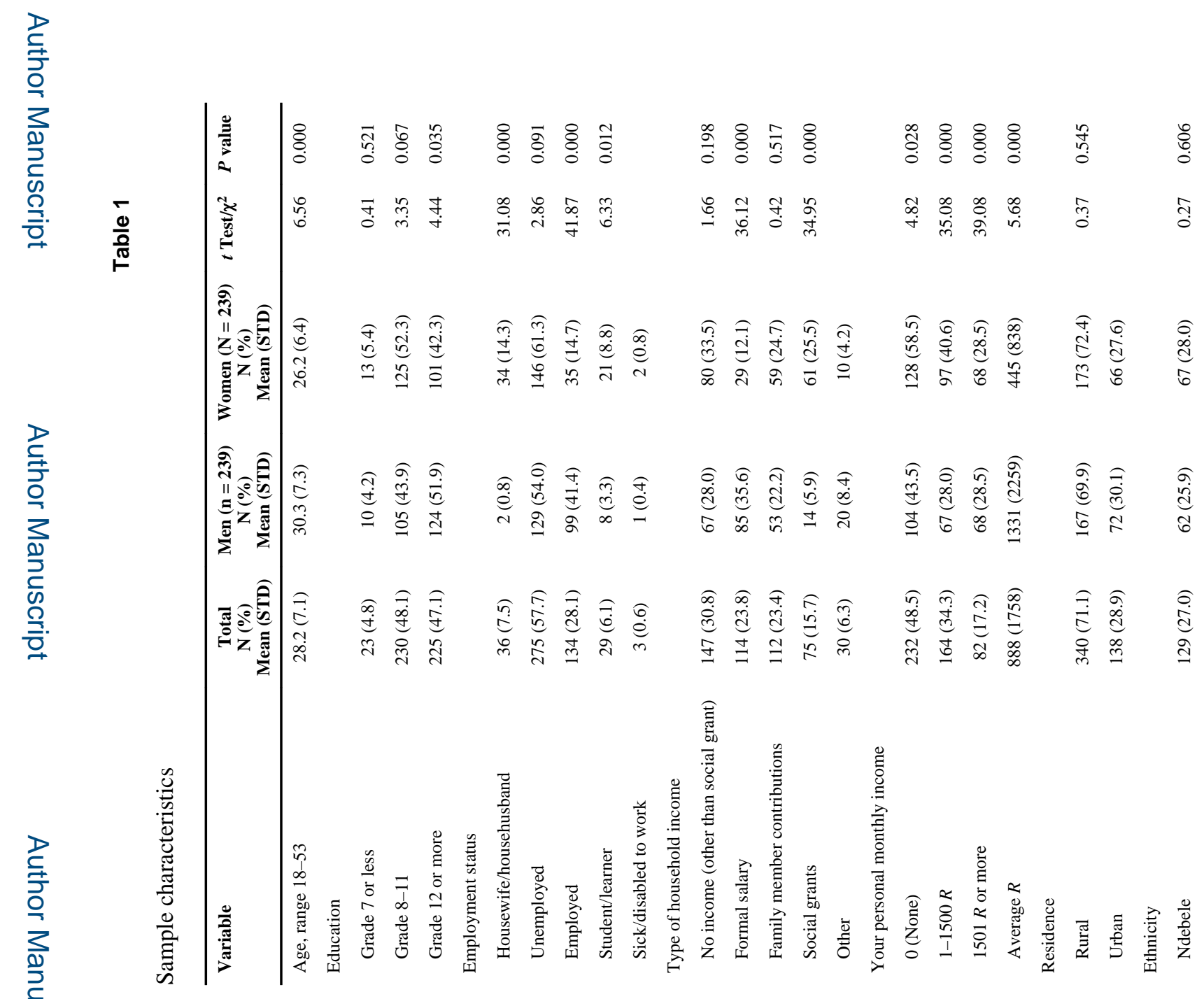




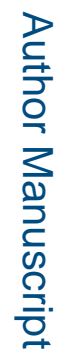

$$
\text { ב্ב }
$$

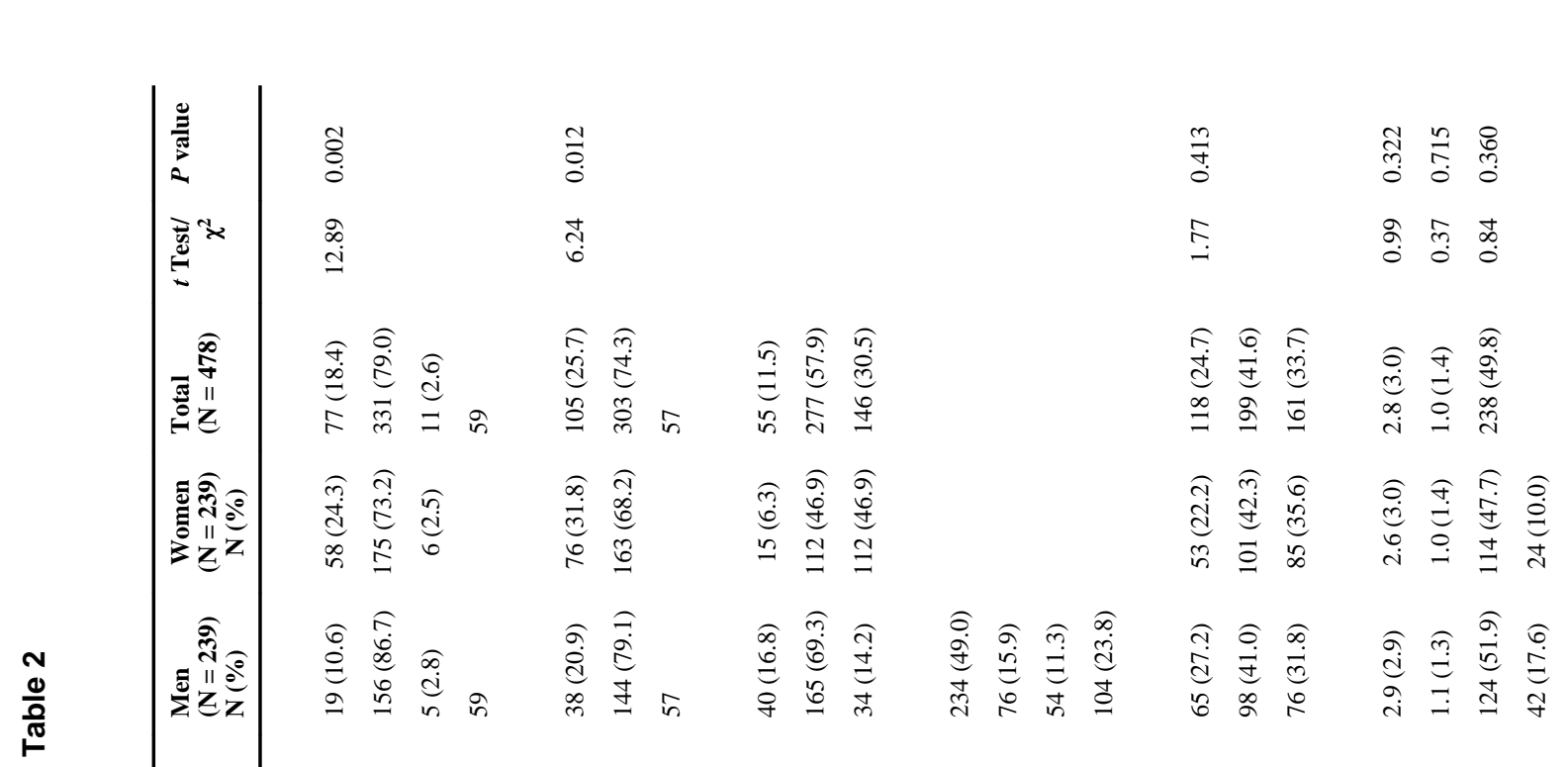

\section{}

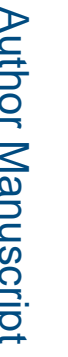




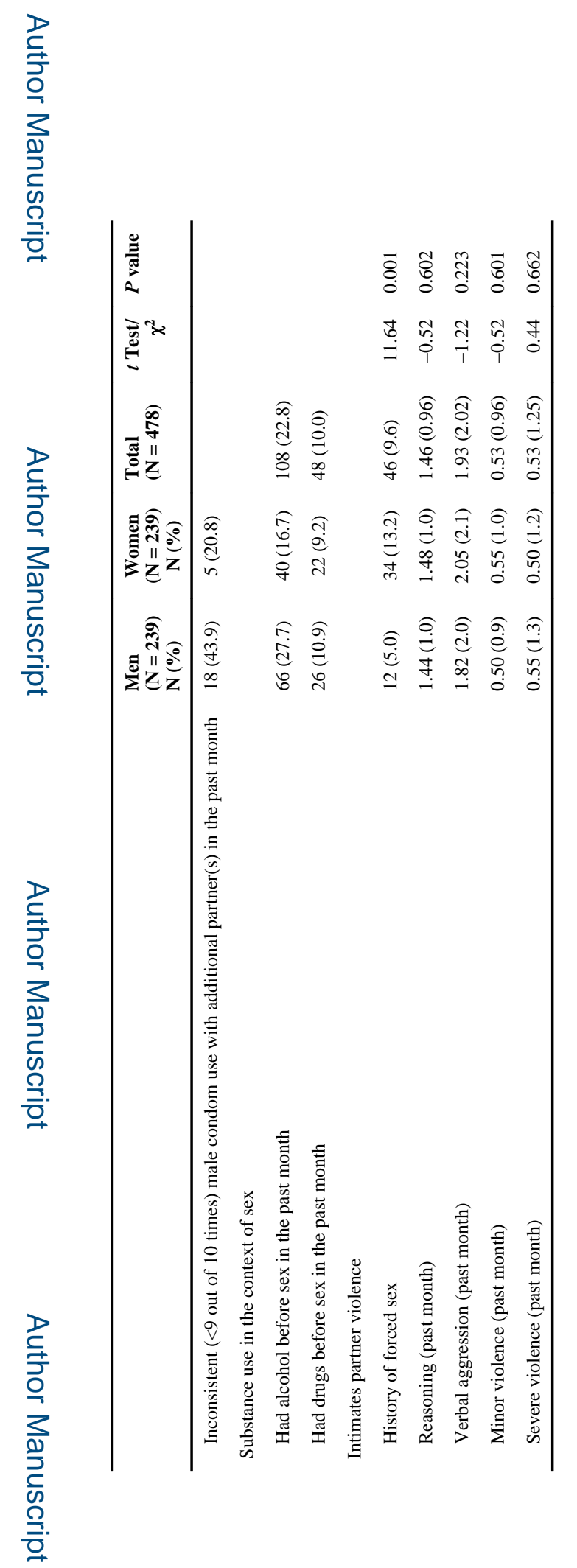


Table 3

Regression model, unprotected intercourse in the past 7 days

\begin{tabular}{|c|c|c|c|c|}
\hline & \multicolumn{2}{|l|}{$\underline{\text { Men }}$} & \multicolumn{2}{|l|}{ Women } \\
\hline & OR $(95 \%$ CI $)$ & $\operatorname{AOR}(95 \% \mathrm{CI})^{a}$ & OR $(95 \% \mathrm{CI})$ & $\operatorname{AOR}(95 \% \mathrm{CI})^{b}$ \\
\hline \multicolumn{5}{|l|}{ Socio-economic factors } \\
\hline Age & $0.97(0.94-1.01)$ & & $0.97(0.93-1.01)$ & \\
\hline \multicolumn{5}{|l|}{ Education } \\
\hline Grade 7 or less & 1.00 & & 1.00 & \\
\hline Grade $8-11$ & $1.42(0.38-5.31)$ & & $1.04(0.33-3.20)$ & \\
\hline Grade 12 or more & $1.88(0.51-7.00)$ & & $1.10(0.35-3.50)$ & \\
\hline \multicolumn{5}{|l|}{ Household income } \\
\hline No income (other than social grant) & 1.00 & & 1.00 & \\
\hline Formal salary & $1.94(0.86-4.35)$ & & $1.03(0.35-3.02)$ & \\
\hline Family member contributions & $1.70(0.70-4.16)$ & & $2.07(0.83-5.18)$ & \\
\hline Social grants & $1.72(0.41-7.27)$ & & $1.69(0.71-4.06)$ & \\
\hline \multicolumn{5}{|l|}{ Residence } \\
\hline Urban & 1.00 & & 1.00 & \\
\hline Rural & $0.81(0.47-1.41)$ & & $0.74(0.42-1.31)$ & \\
\hline \multicolumn{5}{|l|}{ Ethnicity } \\
\hline Ndebele & 1.00 & 1.00 & 1.00 & 1.00 \\
\hline Zulu & $1.44(0.76-2.72)$ & $1.65(0.78-3.48)$ & $1.70(0.95-3.03)$ & $1.93(1.04-3.57)^{*}$ \\
\hline Northern Sotho & $0.90(0.39-2.08)$ & $0.95(0.36-2.46)$ & $1.26(0.57-2.78)$ & $1.25(0.54-2.85)$ \\
\hline Swati & $10.24(1.22-85.78)^{*}$ & $8.39(0.93-75.78)$ & $11.16(2.40-51.94)^{* *}$ & $16.85(3.26-87.13)^{* * *}$ \\
\hline Number of own children & $0.92(0.75-1.13)$ & & $0.99(0.79-1.25)$ & \\
\hline \multicolumn{5}{|l|}{ HIV related variables } \\
\hline \multicolumn{5}{|l|}{ HIV knowledge } \\
\hline Low (4-8) & 1.00 & & 1.00 & \\
\hline Medium (9-10) & $1.26(0.56-2.85)$ & & $0.69(0.27-1.72)$ & \\
\hline $\operatorname{High}(11-12)$ & $1.54(0.67-3.56)$ & & $0.95(0.37-2.41)$ & \\
\hline Self-reported HIV positive & $0.22(0.07-0.70)^{* *}$ & $0.76(0.13-4.44)$ & $0.25(0.13-0.49)^{* * *}$ & $0.33(0.09-1.15)$ \\
\hline HIV positive from medical file & $0.44(0.21-0.93)^{*}$ & $1.00(0.32-3.09)$ & $0.40(0.21-0.76)^{* *}$ & $0.68(0.21-2.19)$ \\
\hline Knows partner is HIV positive & $0.29(0.14-0.62)^{* * *}$ & $0.17(0.05-0.58)^{* *}$ & $0.14(0.03-0.65)^{* *}$ & $0.40(0.08-2.08)$ \\
\hline Had sex with someone else in the past month & $2.10(1.05-4.24)^{*}$ & $3.20(1.29-9.95)^{*}$ & $0.92(0.40-2.15)$ & \\
\hline Alcohol before sex & $0.69(0.39-1.22)$ & & $1.42(0.72-2.81)$ & \\
\hline Drugs before sex & $0.92(0.41-2.08)$ & & $1.65(0.68-4.01)$ & \\
\hline \multicolumn{5}{|l|}{ Intimate partner violence } \\
\hline History of forced sex & $1.32(0.41-4.27)$ & & $1.47(0.71-3.05)$ & \\
\hline Reasoning (past month) & $0.86(0.66-1.13)$ & & $0.89(0.68-1.15)$ & \\
\hline Verbal aggression (past month) & $0.88(0.77-1.00)$ & & $0.90(0.79-1.02)$ & \\
\hline Minor violence (past month) & $0.73(0.55-0.97)^{*}$ & $0.59(0.41-0.85)^{* *}$ & $0.97(0.75-1.26)$ & \\
\hline Severe violence (past 12 months) & $0.81(0.65-1.00)$ & & $0.99(0.80-1.21)$ & \\
\hline
\end{tabular}




\begin{tabular}{|c|c|c|c|c|}
\hline & \multicolumn{2}{|l|}{$\underline{\text { Men }}$} & \multicolumn{2}{|l|}{$\underline{\text { Women }}$} \\
\hline & OR $(95 \%$ CI $)$ & $\operatorname{AOR}(95 \% \mathrm{CI})^{a}$ & OR $(95 \% \mathrm{CI})$ & $\operatorname{AOR}(95 \% \mathrm{CI})^{b}$ \\
\hline \multicolumn{5}{|l|}{ Couple concordance model } \\
\hline \multicolumn{5}{|l|}{ HIV concordance } \\
\hline HIV negative concordant & 1.00 & & 1.00 & \\
\hline HIV positive concordant & $0.31(0.13-0.93)^{* *}$ & & $0.33(0.14-0.79)^{*}$ & \\
\hline HIV discordant & $0.38(0.14-1.06)$ & & $0.21(0.07-0.63)^{* *}$ & \\
\hline
\end{tabular}

${ }^{a}$ Hosmer and Lemeshow $\chi^{2}$ 4.27, $P$ 0.748; Cox and Snell $R^{2} 0.17$; Nagelkerke $R^{2} 0.22$

${ }^{b}$ Hosmer and Lemeshow $\chi^{2}$ 4.81, $P$ 0.569; Cox and Snell $R^{2}$ 0.15; Nagelkerke $R^{2} 0.20$

${ }^{* * *} P<0.001$;

** $P<0.01$;

${ }^{*} P<0.05$ 


\section{Table 4}

Multilevel model, unprotected intercourse in the past 7 days

\begin{tabular}{|c|c|}
\hline & $\begin{array}{l}\text { Couples } \\
\text { B (SE) }(95 \% \text { CI })\end{array}$ \\
\hline \multicolumn{2}{|l|}{ Socio-economic factors } \\
\hline Age & $0.004(0.02),(-0.03$ to 0.03$)$ \\
\hline Education & $0.01(0.07),(-0.13$ to 0.16$)$ \\
\hline Urban versus rural & $0.08(0.06),(-0.03$ to 0.19$)$ \\
\hline \multicolumn{2}{|l|}{ HIV related variables } \\
\hline Self-reported HIV positive & $-0.37(007),(-0.23 \text { to }-0.50)^{* * *}$ \\
\hline HIV positive from medical file & $-0.26(0.06),(-0.38 \text { to }-0.15)^{* * *}$ \\
\hline Knows partner is HIV positive & $-0.35(0.09),(-0.17 \text { to }-0.53)^{* * *}$ \\
\hline Had sex with someone else in the past month & $-0.01(0.08),(-0.16$ to -0.15$)$ \\
\hline Alcohol before sex & $-0.07(0.07),(-0.08$ to -0.21$)$ \\
\hline Drugs before sex & $-0.001(0.09),(-0.18$ to -0.18$)$ \\
\hline \multicolumn{2}{|l|}{ Intimate partner violence } \\
\hline History of forced sex & $-0.04(0.11),(-0.25$ to 0.17$)$ \\
\hline Reasoning (past month) & $0.04(0.12),(-0.19$ to 0.27$)$ \\
\hline Verbal aggression (past month) & $0.06(0.06),(-0.06$ to 0.17$)$ \\
\hline Violence (past month) & $0.05(0.07),(-0.06$ to 0.17$)$ \\
\hline \multicolumn{2}{|l|}{$\begin{array}{l}* * * \\
\quad P<0.001\end{array}$} \\
\hline${ }^{* *} P<0.01$ & \\
\hline
\end{tabular}

